\title{
Outcome of endoscopy-assisted microscopic extended transsphenoidal surgery for suprasellar adult craniopharyngiomas
}

\author{
Hidetoshi lkeda ${ }^{*}$, Hiromi Gotoh ${ }^{2}$ and Kazuo Watanabe ${ }^{3}$ \\ ${ }^{1}$ Research Institute for Pituitary Disease, Southern Tohoku General Hospital, Koriyama, Japan \\ 2 Department of Neurosurgery, Southern Tohoku General Hospital, Koriyama, Japan \\ ${ }^{3}$ Research Institute for Neuroscience, Southern Tohoku General Hospital, Koriyama, Japan
}

\section{Edited by:}

Ken Ho, Garvan Institute of Medical

Research, Australia

Reviewed by:

Roberto Salvatori, Johns Hospital

University, USA

Fahrettin Kelestimur, Erciyes

University, Turkey

*Correspondence:

Hidetoshi Ikeda, Research Institute

for Pituitary Disease, Southern

Tohoku General Hospital, 7-115

Yatsuyamada, Koriyama, Fukushima 963-8563, Japan.

e-mail: ikeda@nsg.med.tohoku.ac.jp
Craniopharyngiomas are difficult to treat. The extended transsphenoidal approach has recently been described in several small series. We describe the usefulness of microscopyassisted angled endoscopy for visualizing vital structures such as tumor attachment or tumor invasion to the pituitary stalk to achieve confident radical tumor removal. Between 2006 and 2010, 15 patients underwent the microscopy-assisted extended transsphenoidal approach for resection of entirely suprasellar craniopharyngiomas. Fourteen patients had the transinfundibular type, and one had the transinfundibular type with extension to the third ventricle. We observed color change within the pituitary stalk by endoscopy. The pituitary stalk was cut intentionally in 10 patients because of suspected tumor invasion surrounding the stalk. Total removal was accomplished in nine patients. Pathological specimens from the pituitary stalk showed tumor invasion spreading over the surface of the pituitary stalk, shown by a discolored pituitary stalk, and this was essential for confident radical tumor removal. Even after stalk resection, postoperative diabetes insipidus was minimal when a bright signal on T1 in the posterior lobe was not observed with preoperative magnetic resonance imaging. Confident radical tumor removal is possible with the introduction of the endoscopy-assisted microscopic extended transsphenoidal approach.

Keywords: craniopharyngioma, extended transsphenoidal, endoscopy, pituitary stalk, surgical approach, endoscope, total removal

\section{INTRODUCTION}

Various approaches including the transcranial and transsphenoidal routes (Suzuki et al., 1984; Koenig et al., 1986; Hoffman et al., 1992; Maira et al., 1995) have been proposed for removal of craniopharyngiomas. Although there is consensus on the use of transsphenoidal surgery (TSS) for purely intrasellar craniopharyngiomas (Koenig et al., 1986), extended TSS, originally described by Kato et al. (1998), provides excellent midline access and visibility of the suprasellar space, while obviating brain and optic nerve retraction (Dumon et al., 2006). In subsequent years, several authors have described treatment of craniopharyngiomas with microscopy-assisted supra-diaphragmatic extended TSS (Maria et al., 2004; Chakrabarti et al., 2005; Dusick et al., 2005; Laws et al., 2005). Based on the surgical experience of more than 1500 cases of the transsphenoidal approach, we introduced endoscopy-assisted extended TSS for suprasellar craniopharyngiomas in 2006.

Despite the benign histological appearance of these tumors, they are a major challenge for the neurosurgeon because of their tendency to adhere to vital structures such as the optic apparatus, pituitary stalk, hypothalamus, and third ventricle (Laws et al., 2005). The transcranial approach (Suzuki et al., 1984) is insufficient for providing a wide scope of exposure for craniopharyngiomas that originate from the pituitary stalk or upper surface of the pituitary gland. Therefore, tumor removal is usually insufficient because of the limited space of manipulation and limited view scope of the origin of the craniopharyngioma. The endoscopy-assisted extended transsphenoidal approach has the advantage of identifying the origin of the craniopharyngioma and preserving the blood supply for the optic chiasm and pituitary stalk by applying microsurgical methods and careful dissection.

The ideal approach for suprasellar craniopharyngiomas has been extensively investigated (Hoffman et al., 1992; Steno et al., 2004). The recent application of endoscopy to TSS has encouraged some surgeons with experience in endoscopic pituitary surgery to perform removal of suprasellar lesions by using pure, fully endoscopic, extended transnasal TSS (Jho and Carrau, 1997; Gardner et al., 2008; Kassam et al., 2008). This technique enables panoramic visualization of the surgical target and its relevant anatomical structures. However, endoscopy-assisted microscopic extended TSS has definite advantages with regard to surgical maneuverability during tumor dissection and sellar floor reconstruction. We present our experience with endoscopy-assisted microscopic extended TSS for 15 patients with entirely suprasellar craniopharyngiomas [i.e., transinfundibular type: type II, Kassam classification (Kassam et al., 2008)], and we evaluated the tumor removal rate, visual recovery, and postoperative neuro-endocrine function. In addition, we investigated the pathological features of 
stalk specimens that were suspected of tumor invasion by endoscopic inspection, as well as endocrinological changes before and after resection of the stalk.

\section{MATERIALS AND METHODS PATIENT POPULATION}

Fifteen patients who had undergone extended TSS for craniopharyngioma at Ohara Medical Center Hospital between 2006 and 2007 and at Southern Tohoku General Hospital between 2008 and 2010 were examined. We used endoscopy (Olympus, OME8000 during 2006 and 2007; Olympus, Endoarm during 2008 through 2010) for the assistance of microsurgery. Extended TSS was performed by only one skilled pituitary neurosurgeon (H.I.). Perioperative therapy and management were carried out according to a clinical protocol of the hospital. The age of the patients ranged from 38- to 76-years-old (mean age, 58-years-old). Nine patients were female and six patients were male. Informed consent was obtained from all patients.

Thirteen out of 15 patients had not undergone previous surgery or other therapy, one patient had undergone both transcranial surgery and gamma knife surgery, and the other patient had undergone transcranial surgery twice.

\section{NEUROIMAGING EVALUATION}

The size and extent of the tumor were determined using magnetic resonance (MR) images obtained with a $1.5 \mathrm{~T}$ system (Magnetome, Siemens, Erlangen, Germany) and 3.0 T MR units (Achieve Quasar Dual 3.0 T, Philips, Netherlands). T1- and T2-weighted thick sagittal and coronal spin-echo MR images were obtained as 2- to 3-mmthick slices. Additional T1-weighted axial, sagittal, and coronal MR images (500/15 ms) were obtained immediately after injection of $0.1 \mathrm{mmol} / \mathrm{kg}$ gadolinium-diethylenetriaminepenta-acetic acid (Schering, Berlin, Germany). Images were reconstructed by two-dimensional Fourier transformation on a $256 \times 256$ image matrix.

\section{ENDOCRINOLOGICAL ASSESSMENT}

The endocrine status of the patients was evaluated before and after surgery by measurement of serum levels of growth hormone $(\mathrm{GH})$, insulin-like growth factor-1 (IGF-1), prolactin (PRL), adrenocorticotropic hormone (ACTH), cortisol, thyroid-stimulating hormone (TSH), free-T3 (triiodothyronine) and free-T4 (thyroxine), follicle-stimulating hormone (FSH), luteinizing hormone $(\mathrm{LH})$, estradiol, and free testosterone. Precise endocrinological tests, such as the (corticotropin-releasing hormone) + (thyrotropinreleasing hormone $)+($ LH-releasing hormone) loading test and the GHRP-2 loading test, were carried out if necessary.

\section{PATHOLOGICAL EXAMINATION}

Tumor specimens were fixed immediately after removal. Specimens were fixed in $10 \%$ neutral buffered formalin. The samples were then embedded in paraffin and sections of $3 \mu \mathrm{m}$ thickness were cut. They were used for hematoxylin and eosin staining and immunohistochemical staining by the $\mathrm{ABC}$ method using the following antibodies: polyclonal ACTH (DAKO, Tokyo, Japan), polyclonal GH (DAKO), polyclonal PRL (DAKO), monoclonal TSH-beta (Neo markers, CA, USA), monoclonal LH-beta (Neo markers), monoclonal FSH-beta (Neo markers), polyclonal alphasubunit (DAKO), cytokeratin (DAKO), S-100 protein (DAKO), synaptophysin (DAKO), and monoclonal Ki-67 (DAKO). Ki-67labeled cells were determined by counting the number of positive cells in more than 1000 tumor cells in at least four representative high power fields $(\times 400)$ across the slide.

\section{OPHTHALMOLOGICAL ASSESSMENT}

The visual acuity and visual field were evaluated before and after surgery. The visual field was tested with the Goldmann visual field and Hanfry visual field.

\section{EVALUATION OF TUMOR REMOVAL}

The tumor was considered to be totally resected when the extent of the resection met the following criteria: (1) there was no suspicious tumor on intraoperative endoscopic inspection, and (2) there was no remnant of the tumor on 2 or 3 month post-operative MR imaging. A subtotal resection was when at least $50 \%$ of the tumor was removed, and a partial resection was when more than $50 \%$ of the tumor remained.

\section{RESULTS}

\section{CLINICAL AND RADIOLOGICAL PRESENTATION}

During operative findings of the pituitary stalk, all patients had a transinfundibular tumor (type 2: Kassam classification; Gardner et al., 2008). If the tumor had a small component within the sella but had not expanded to the bony confines of the sella, the tumor was considered suprasellar. All of the tumors were entirely suprasellar in location and firmly connected to the pituitary stalk, suggesting that the origin of the tumor was the pituitary stalk. The maximal tumor diameter was measured on preoperative MR imaging studies and was measured as the largest diameter in the coronal, axial, or sagittal planes. Tumors were categorized as cystic, solid, or mixed based on their predominant features. Eight patients had mainly cystic tumors, five had mixed cystic and solid tumors, and two had solid tumors (Table 1).

\section{SURGICAL APPROACH}

All patients underwent endoscopy-assisted microscopic sublabial extended TSS. At the beginning of surgery, we set up lumbar drainage to accommodate cerebrospinal fluid (CSF) flow during the operation. The extended transsphenoidal approach enabled us to observe proper orientation of the surgical anatomical view, and to observe important structures such as the optic nerve, oculomotor nerve, the internal carotid artery, the basilar artery, and the anterior communicating artery. By dissecting tumor feeder vessels and the tumor capsule from the inferior and posterior surface of the optic chiasm under microscopic view, nutrient arteries from the superior hypophyseal artery to the optic chiasm were preserved as much as possible. Assisted endoscopy also enabled a clear view of the interrelationship between the pituitary stalk and tumor attachment. Even the dead angle when only observing with the microscope, such as behind the pituitary stalk and the upper surface of the pituitary gland, was clearly visualized with endoscopic assistance. In particular, we noted the color of the whole pituitary stalk, since it is possible to determine invasion of a 
Table 1 | Clinical and radiological findings in 15 patients with craniopharyngioma treated using endoscopy-assisted extended microscopic TSS.

\begin{tabular}{|c|c|c|c|c|c|c|c|c|}
\hline No. & Age & Sex & Previous treatment & Kassam classification & $\begin{array}{l}\text { Solid or } \\
\text { cystic }\end{array}$ & Calcification & $\begin{array}{l}\text { Pre-operative } \\
\text { DI }\end{array}$ & $\begin{array}{l}\text { T1 high signal of } \\
\text { posterior lobe }\end{array}$ \\
\hline 1 & 43 & $\mathrm{~F}$ & None & Transinfundibular (type II) & Cystic & - & No & + \\
\hline 3 & 57 & M & None & Transinfundibular (type II) & Mixed & - & Yes & - \\
\hline 4 & 56 & $\mathrm{~F}$ & None & Transinfundibular (type II) & Mixed & ++ & No & - \\
\hline 5 & 59 & $\mathrm{~F}$ & Craniotomy + gamma & Transinfundibular (type II) & Cystic & + & Yes & - \\
\hline 7 & 66 & M & None & Transinfundibular (type II) & Solid & - & No & - \\
\hline 8 & 37 & M & Craniotomy (two times) & Transinfundibular (type II) & Cystic & - & Yes & - \\
\hline 9 & 72 & $\mathrm{~F}$ & None & Transinfundibular (type II) & Cystic & + & No & + \\
\hline 10 & 76 & M & None & Transinfundibular (type II) + III ventricle & Cystic & + & No & - \\
\hline 11 & 67 & M & None & Transinfundibular (type II) & Cystic & + & No & - \\
\hline
\end{tabular}

craniopharyngioma to the pituitary stalk and make a decision on whether to cut it depending on a slight change of color or shape. Therefore, we can achieve a more confident radical resection of the tumor by introduction of endoscopy-assisted microscopic TSS.

\section{SURGICAL OUTCOME}

By precise endoscopic observation of the pituitary stalk, we decided to cut the pituitary stalk in 10 out of 15 cases to totally remove the tumor. Total removal of the tumor was achieved in nine patients (Figures 1A,B), five had subtotal resection, and one had partial removal because of a rapid pathological diagnosis of squamous cell carcinoma (Table 2). None of the cases showed CSF fistula or neurological deficit after surgery.

\section{PATHOLOGICAL RESULTS}

Histological characteristics of the craniopharyngiomas included the adamantinomatous type in eight out of 15 specimens, six specimens were the papillary type, and one specimen was malignant craniopharyngioma (Table 2 ). In the case where the pituitary stalk was intentionally cut, pathological study revealed that the epithelial nature of the craniopharyngioma tended to grow surrounding the whole surface of the pituitary stalk in all seven cases examined (Figure 2).

\section{VISUAL OUTCOMES}

Details of pre- and post-operative visual acuity and the visual field are summarized in Table 3 and Figure 3. Visual fields were either improved or unchanged in all patients. With regard to visual acuity, 26 out of 30 eyes were improved after surgery.

\section{ENDOCRINE OUTCOMES}

Details of pre- and post-operative hormone replacement therapy are summarized in Table 4. Although 10 patients needed to have the pituitary stalk cut because of a highly suspicious appearance of tumor invasion around the pituitary stalk during endoscopic observation, all of the cases, except for one case who showed diabetes insipidus (DI) before surgery, did not show DI after surgery. Severe GH deficiency can be diagnosed with high reliability using $15 \mu \mathrm{g} / \mathrm{l}(9 \mu \mathrm{g} / \mathrm{l}$ when $\mathrm{GH}$ is calibrated with the recombinant World Health Organization 98/574 standard) as the cut-off peak of GH concentration (Chihara et al., 2007). Twelve out of 15 patients fulfilled this criteria of adult severe GH deficiency (Billar et al., 2002; Chihara et al., 2007). However, there were nine out of 12 patients $(75 \%)$ patients who showed falsenegative IGF-1 SD scores for severe adult GH deficiency. This is in contrast to patients with pituitary adenoma who fulfilled the criterion for adult severe GH deficiency, because six of these 36 patients with pituitary adenoma (17\%) showed false-negative IGF-1 SD scores for severe adult GH deficiency (data not shown). Therefore, most of the patients with craniopharyngioma showed a normal range of IGF-1 values in spite of severe adult GH deficiency.

When the pituitary stalk was completely cut, it was evaluated for the secretory status of pituitary hormone. Details of changes in pre- and post-operative serum hormone values are summarized in Table 4. FSH/LH levels were measured in female patients. Free testosterone levels were measured in male patients, and mean values were below normal both before and after stalk resection.

In patients with pituitary stalk resection, serum ACTH, TSH, $\mathrm{LH}$, and FSH levels were decreased ( $p<0.05$; Wilcoxon signedranks test) after the operation, while serum GH, PRL, and IGF-1 levels either remained unchanged or were slightly increased after the operation (Figure 4). Even if GH and IGF-1 levels were within normal limits after surgery, patients with stalk resection inevitably became adult GH deficient based on the results of a GHRP-2 loading test for GH secretion. On the other hand, those patients without DI did not show permanent DI preoperatively even after resection of the pituitary stalk during the operation. We did not observe permanent DI after resection of the pituitary stalk in patients whose three-dimensional MRI did not show a bright T1 


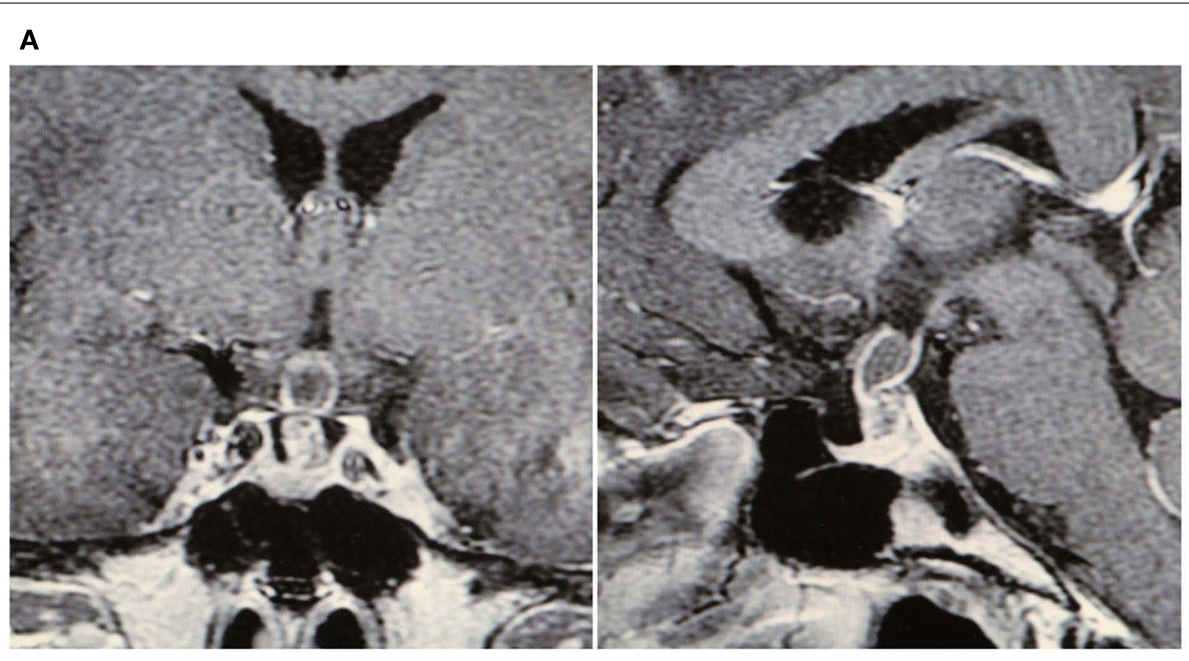

B

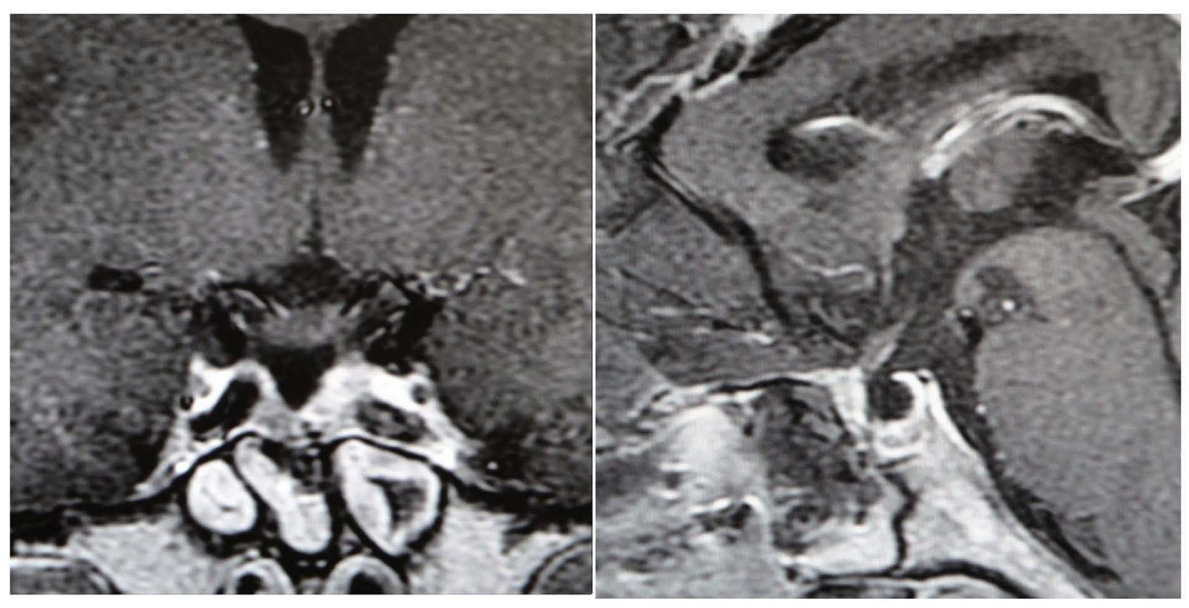

FIGURE 1 | (A) Pre-operative and MRI of a 60-year-old female (case 6). (B) Postoperative MRI.

Table 2 | Surgical outcome.

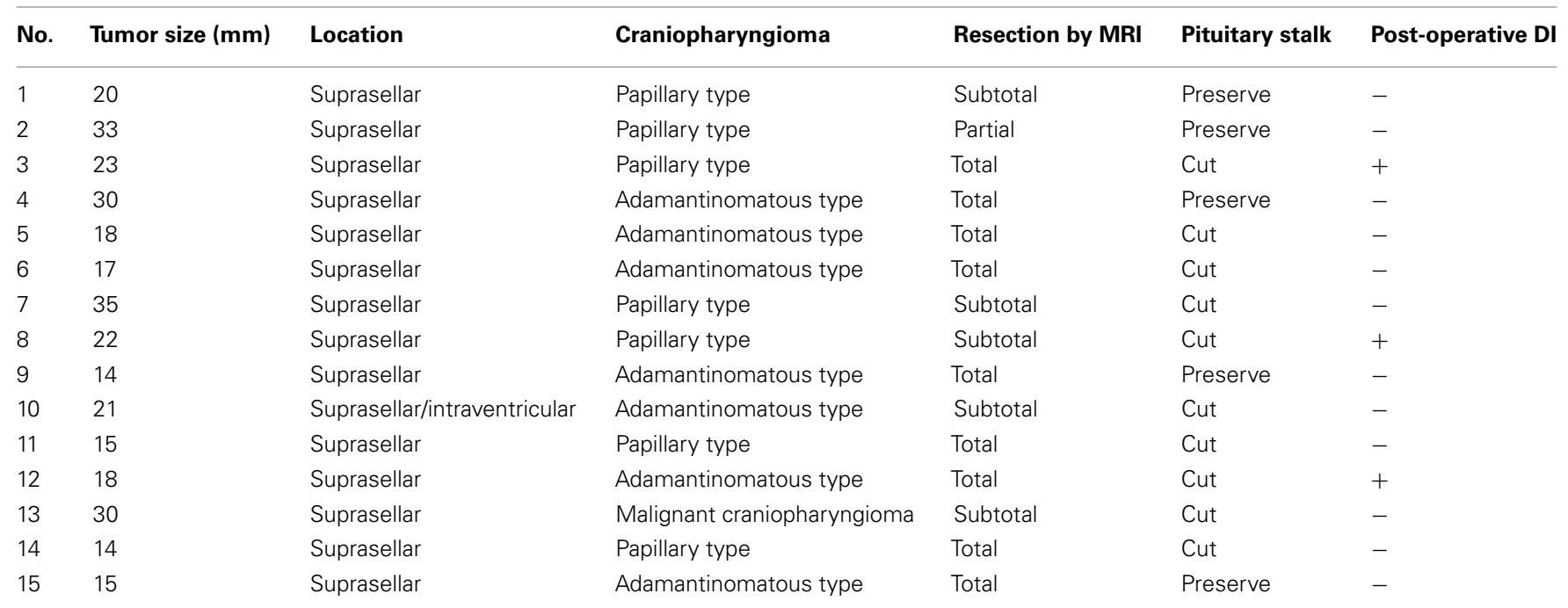



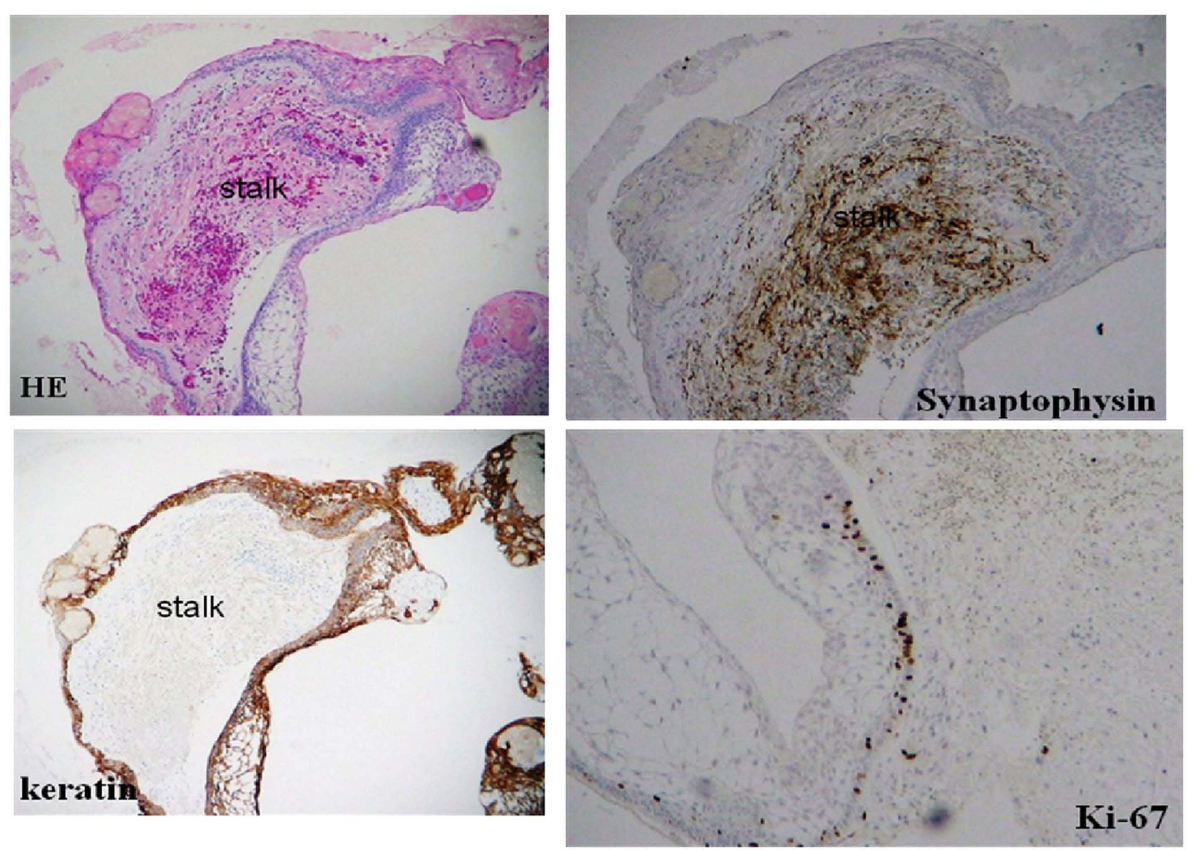

FIGURE 2 | Pathological findings of a resected tumor including the pituitary stalk (case 6).

Table 3 | Visual field before and after the operation.

\begin{tabular}{lll}
\hline Case no. & Pre-operative VF & Post-operative VF \\
\hline 1 & BTH & Full \\
2 & BTH & Improved \\
3 & BTH & Full \\
4 & BTH & BTH \\
5 & Full & Full \\
6 & Full & Full \\
7 & BTH & Improved \\
8 & BTH & Improved \\
9 & Full & Full \\
10 & BTH & Full \\
11 & BTH & Full \\
12 & Full & Full \\
13 & BTH & Improved \\
14 & Full & Full \\
15 & Full & Full \\
\hline
\end{tabular}

VF, visual field; BTH, bitemporal hemianopsia; full, normal visual field.

signal in the posterior lobe on preoperative superconductive MRI (Table 1).

\section{ADJUVANT THERAPY}

A follow-up MRI showed no tumor recurrence in all of the nine patients in whom total removal was achieved. Five out of 15 patients were treated with gamma knife surgery after the operation and were followed up without tumor growth. One patient with partial removal was conventionally irradiated because the residual tumor was too large for gamma knife surgery.

\section{DISCUSSION}

Craniopharyngiomas are histologically benign, but they are a major challenge for the neurosurgeon because of their tendency to adhere to vital structures such as the optic apparatus, the pituitary stalk, the hypothalamus, and the third ventricle. In cases in which gross total removal is the goal, the difficulty of dealing with the blood supply of the tumor and its possible adherence to these important structures must be considered.

Suprasellar craniopharyngiomas have been approached via various transcranial routes, preferably including the interhemispheric (Suzuki et al., 1984), pterional (Maira et al., 1995), and subfrontal approaches. However, the transcranial approach has several disadvantages, including the need for brain retraction and manipulation of many neurovascular structures. In addition, the inferior and posterior surfaces of the optic nerves, which are the most common sites to which craniopharyngiomas usually adhere, cannot be visualized directly with a microscopic view when a transcranial trajectory is used. Therefore, a degree of blind dissection is unavoidable, which may result in incomplete removal of the tumor or injury to the optic nerve and fine perforating vessels (Wang et al., 2002). Accessibility is particularly limited in the transcranial approach when the optic chiasm is prefixed or if the tumor is mainly located behind the optic chiasm.

The TSS approach has many advantages compared with various transcranial approaches. Because the trajectory of this approach is usually the same as that of tumor growth, only minimal brain retraction is needed. The surgeon can also identify perforating vessels and separate them from the tumor capsule in the early stage of the operation. This approach also has an advantage regarding optic nerve manipulation. Because internal debulking and capsular dissection are initiated at the lower part of the tumor mass and 


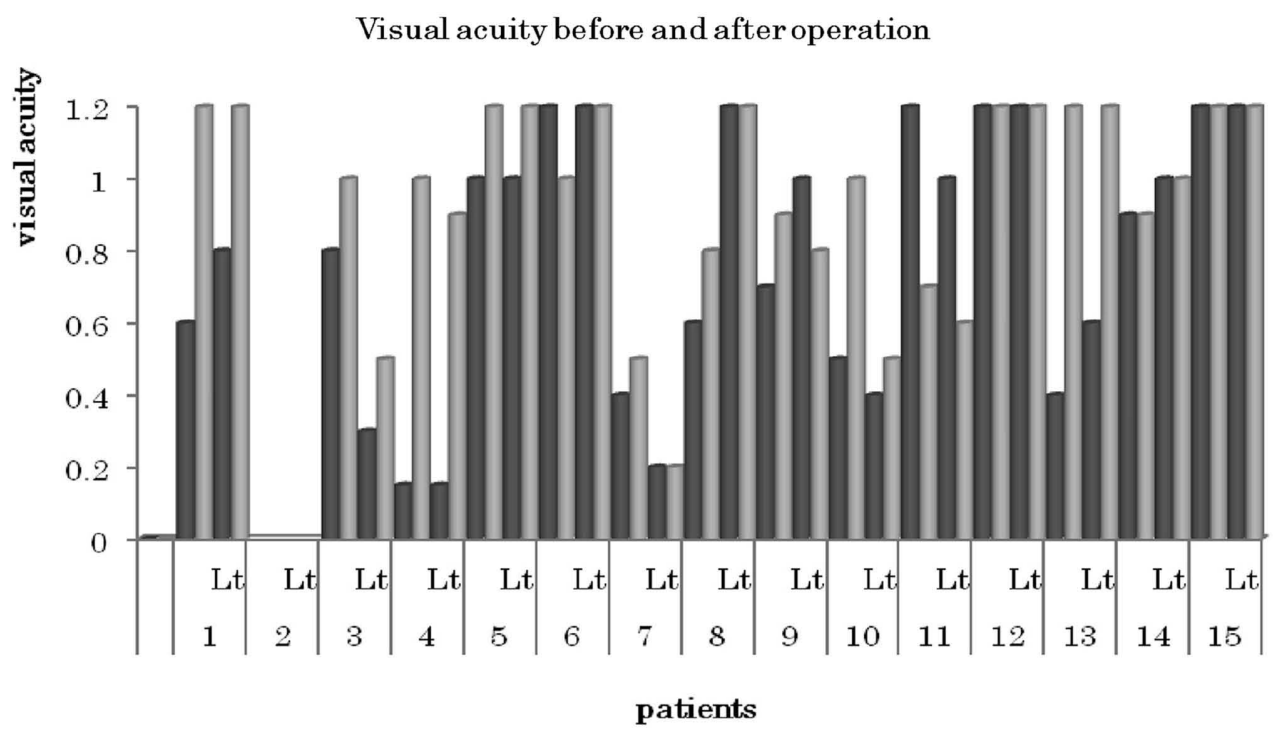

FIGURE 3 | Visual acuity before and after the operation. Black bars: preoperative visual acuity; gray bars: postoperative visual acuity.

Table 4 | Pre-and post-operative hormone supplement.

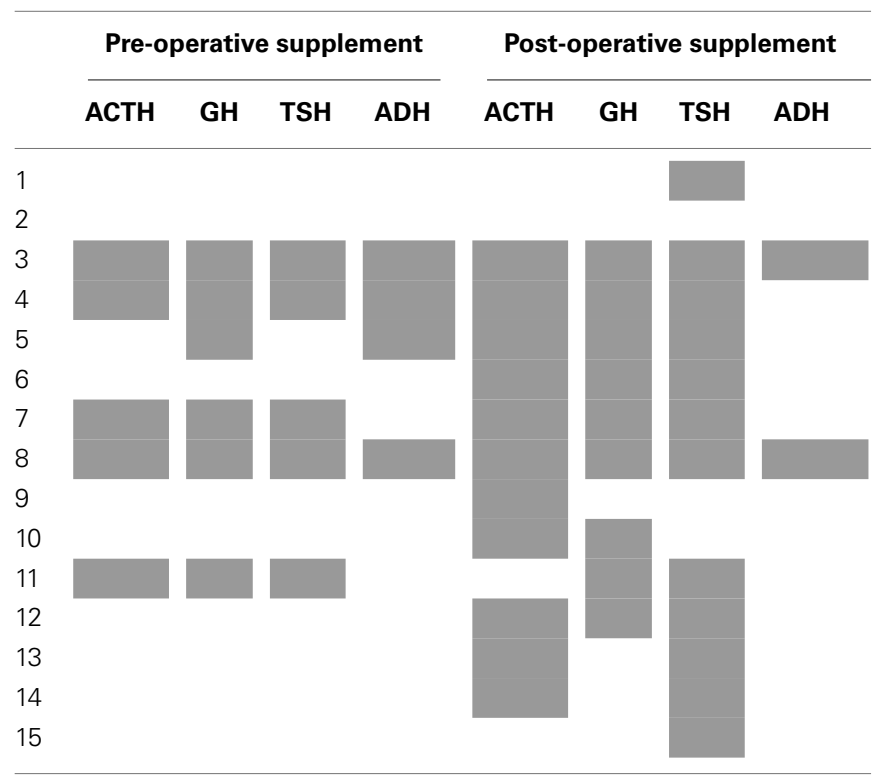

Black columns: replacement therapy(+).

progress to the upper part, the optic nerve can be decompressed without excessive manipulation of neurovascular structures, and because it descends with the upper part of the tumor, separation of the tumor capsule from the optic nerve can be performed with the aid of a direct microscopic view. Furthermore, this approach is less likely to leave a remnant tumor mass or injure fine perforating vessels. Some investigators have achieved encouraging results with extended TSS for the surgical removal of suprasellar craniopharyngiomas (Maria et al., 2004; Chakrabarti et al., 2005; Dusick et al., 2005; Laws et al., 2005; Gardner et al., 2008). They concluded that TSS is not an alternative procedure for such tumors, but it should be the ideal approach. In craniopharyngioma surgery, the main surgical field of interest is limited to a small area near the sellar and suprasellar spaces. Therefore, a panoramic view (Jho and Carrau, 1997; Kassam et al., 2008), which is one of the greatest advantages of an endoscope, is not essential at this step. However, we found that the angled endoscope was very helpful when we needed to visualize spaces that could not be seen with a microscopic view. The endoscope was introduced to resolve any uncertainties during the dissection. It always provided critical information on which part of the tumor to dissect first, which neurovascular structures the tumor adhered to, and where the perforating vessels were located. For the dissection of perforating vessels from the tumor capsule, the most critical procedure during the operation, we preferred the microscopic to the endoscopic view, because we believe that these fine procedures should be performed with a comfortable and maneuverable space for microsurgical instruments since bipolar forceps are required at various angles for dissection of perforating vessels. In addition, the speculum, which is an unusual instrument for an endoscopic procedure, provided sufficient space to introduce the bipolar coagulator and microscissors, and this would have been very difficult without a speculum.

We believe that the best candidates for extended TSS are those who have a tumor with a prefixed optic chiasm or retrochiasmatic growth involving the floor of the third ventricle or the interpeduncular cistern. For these reasons, preoperative evaluation of the relative location of the optic chiasm is important to determine whether the tumor can be properly accessed by TSS.

By observing the color change of the whole pituitary stalk, this can lead to suspected invasion of craniopharyngioma to the pituitary stalk. If there is a slight color change of the pituitary stalk, it is important to cut the stalk with the tumor to achieve confident radical resection of the tumor. This results in a wide operative field so that fine dissection of neurovascular structures from the tumor capsule can be performed efficiently and safely. 


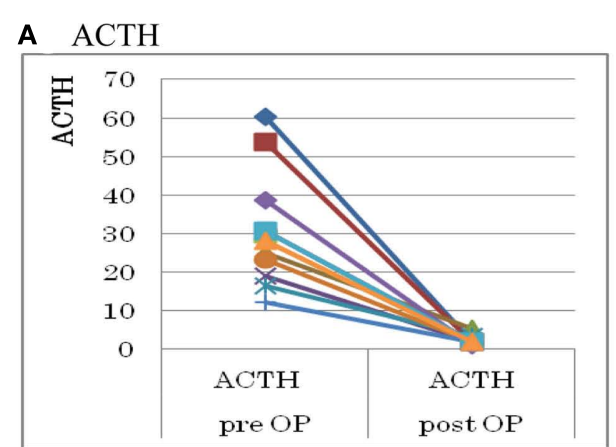

C IGF-1

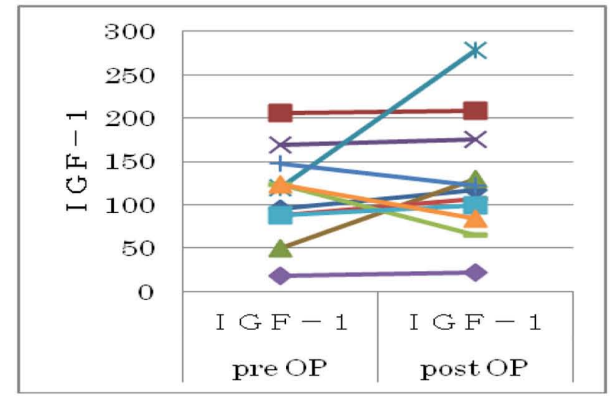

E LH for female patients

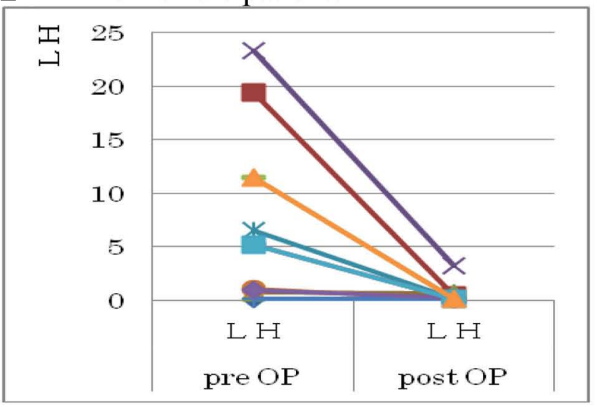

G $\mathrm{GH}$

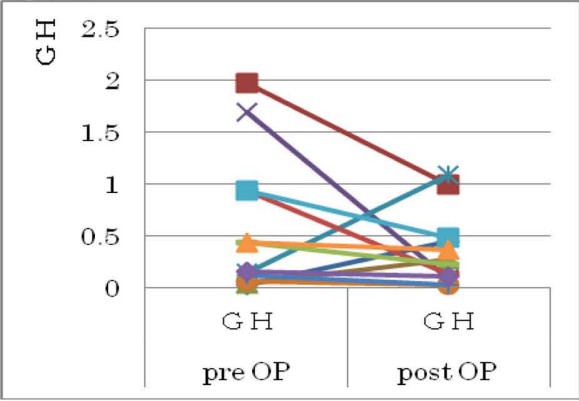

B PRL

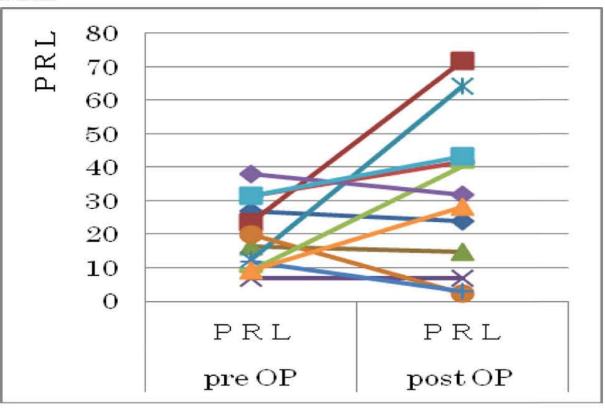

D TSH

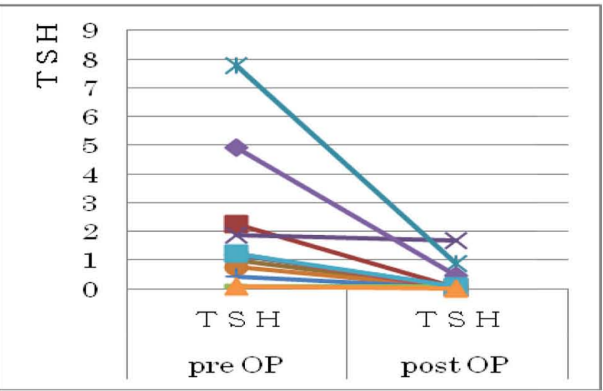

F FSH for female patients

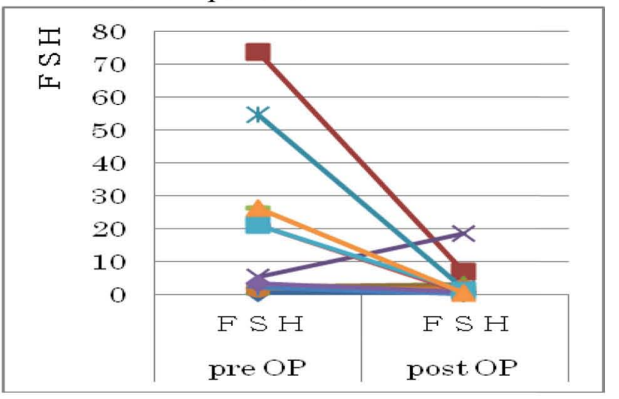

FIGURE 4 | Changes in basal levels of pituitary hormones before and after stalk resection.

Our surgical experience revealed that in those patients whose pituitary stalk was invaded by the tumor, the appearance of permanent DI by pituitary stalk resection was minimal. This is probably due to the compensation phenomenon of the posterior lobe forming a "pseudo-posterior lobe" in the upper part of the stalk (Fujisawa et al., 1987). Patients with a pseudo-posterior lobe will not develop DI because of the presence of vasopressin-producing cells (Kurokawa et al., 1988). Serum levels of IGF-1 will not be decreased in most patients with pituitary stalk resection. However, normal IGF-1 levels do not guarantee normal GH secretion, but GH stimulation tests reveal severe $\mathrm{GH}$ deficiency in almost all cases. Therefore, a prompt GH stimulation test after surgery is essential to determine whether there is severe GH deficiency (Chihara et al., 2007), and it is important to start GH supplementation as soon as possible if total removal of the tumor has been accomplished. Serum ACTH and TSH levels diminish rapidly after stalk resection, 
and therefore supplementation of these hormones is essential just after the operation.

Recently, self-formation of a functional adenohypophysis in three-dimensional culture has been reported (Suga et al., 2011). Therefore, our data provide useful information on whether it is necessary to perform a simultaneous operation to transplant the adenohypophysis, derived from human ES induced pluripotent stem cells, into the cavity just after total removal of the tumor.

In the current study, tumor cells tended to grow superficially surrounding the pituitary stalk; therefore, morphologically intactlooking stalks may have been invaded. This suggests that unless faintly colored stalks are cut, the tumor will recur soon after the operation because of the high Ki-67 labeling index within the invaded tumor. We believe that the advantages of sacrificing the pituitary stalk (i.e., maximizing maneuverability and minimizing the possibility of neurovascular injury), especially with the transinfundibular type, override the risk of tumor recurrence.

\section{CONCLUSION}

Ideal treatment for craniopharyngioma is total removal. However, the suprasellar location interferes with the neurosurgeon's operative view. It is essential to observe stalk attachment of craniopharyngiomas to perform confident total removal. We achieved very encouraging results with endoscope-assisted extended TSS for removing suprasellar craniopharyngiomas with a wider and more precise operative field.

\section{REFERENCES}

Billar, B. M., Samuels, M. H., Zagar, A., Cook, D. M., Arafah, B. M., Bonert, V., Stavrou, S., Kleinberg, D. L., Chipman, J. J., and Hartman, M. L. (2002). Sensitivity and specificity of six tests for the diagnosis of adult $\mathrm{GH}$ deficiency. J. Clin. Endocrinol. Metab. 87, 2067-2079.

Chakrabarti, I., Amar, A. P., Couldwell, W., and Weiss, M. H. (2005). Long-term neurological, visual and endocrine outcomes following transnasal resection of craniopharyngioma. J. Neurosurg. 102, 650-657.

Chihara, K., Shimazu, A., Hizuka, N., Tanaka, T., Seino, Y., and Kato, Y. (2007). A simple diagnostic test using GH-releasing peptide2 in adult GH deficiency. Eur. J. Endocrinol. 157, 19-27.

Dumon, A. S., Kanter, A. S., Jane, J. A., and Laws, E. R. (2006). "Extended transsphenoidal approach," in Pituitary Surgery-A Modern Approach, Vol. 34. eds E. R. Laws and J. P. Sheehan (Basel: Karger), 29-45.

Dusick, J. R., Esposito, F., Kelly, D. F., Cohan, P., DeSalles, A., Becker, D. P., and Martin, N. A. (2005). The extended direct endonasal transsphenoidal approach for nonadenomatous suprasellar tumors. $J$. Neurosurg. 102, 832-841.

Fujisawa, K., Kikuchi, K., Nishimura, K., Togashi, K., Itoh, K., Noma, S., Minami, S., Sagoh, T., Hiraoka, T., and Momoi, T. (1987). Transection of the pituitary stalk: development of an ectopic posterior lobe assessed with MR imaging. Radiology 165 487-489.

Gardner, P. A., Kassam, A. B., Snyderman, C. H., Carrau, R. L., Mintz, A. H., Grahovac, S., and Stefko, S. (2008). Outcomes following endoscopic, expanded endonasal resection of supraseller craniopharyngioma: a case series. J. Neurosurg. 109, 6-16.

Hoffman, H. J., De Silva, M., Humphreys, R. P., Drake, J. M., Smith, M. L., and Blaser, S. I. (1992). Aggressive surgical management of craniopharyngiomas in children. $J$. Neurosurg. 76, 47-52.

Jho, H. D., and Carrau, R. L. (1997). Endoscopic pituitary surgery; an early experience. Surg. Neurol. 47, 213-223.

Kassam, A. B., Gardner, P. A., Snyderman, C. H., Carrau, R. L., Mintz, A. H., and Prevedello, D. M. (2008). Expanded endonasal approach, a fully endoscopic transnasal approach for the resection of midline suprasellar craniophryngiomas: a new classification based on the infundibulum. J. Neurosurg. 108, 715-728.

Kato, T., Sawamura, Y., Abe, H., and Nagashima, M. (1998). Transsphenoidal-transtuberculum sellae approach for supradiaphragmatic tumors: technical note. Acta Neurochir. (Wien) 40, 715-718.

Koenig, A., Luedecke, D. K., and Herrmann, H. D. (1986). Transnasal surgery in the treatment of craniopharyngiomas. Acto Neurochir. (Wien) 3, 1-7.

Kurokawa, H., Fujisawa, I., Nakano, Y., Kimura, H., Akagi, K., Ikeda, K., Uokawa, K., and Tanaka, Y. (1988) Posterior lobe of the pituitary gland: correlation between signal intensity on T1-weighted MR images and vasopressin. Radiology 207, 79-83.

Laws, E. R., Kanter, A. S., Jane, J. A. Jr., and Dumont, A. S. (2005). Extended transsphenoidal approach. J. Neurosurg. 102, 825-828.

Maira, G., Anile, C., Rossi, G. F., and Colosimo, C. (1995). Surgical treatment of craniopharyngiomas: an evaluation of the transsphenoidal and pterional approaches. Neurosurgery 6, 715-724.

Maria, G., Anmile, C., Albanese, A., Cabezas, D., Pardi, F., and Vignati, A. (2004). The role of transsphenoidal surgery in the treatment of craniopharyngiomas. J. Neurosurg. 100, 445-451.

Steno, J., Malacek, M., and Bizik, I. (2004). Tumor-third ventricular relationship in supradiaphragmatic craniopharyngiomas: correlation of morpholofical, magnetic resonance imaging, and operative findings. Neurosurgery 54, 1051-1060.

Suga, H., Kadoshima, T., Minaguchi, M., Ohgushi, M., Soen, M., Nakano, T., Takata, N., Wataya, T., Muguruma, K., Miyoshi, S., Yonemura, S. Oiso, Y., and Sasai, Y. (2011). Selfformation of functional adenohypohysis in three-dimensional culture. Nature 480, 57-62.
Suzuki, J., Katakura, R., and Mori, T. (1984). Interhemispheric approach through the lamina terminalis to tumors of anterior part of the third ventricle. Surg. Neurol. 2, 157-163.

Wang, K. C., Kim, S. K., Choe, G., Chi, J. S., and Cho, B. K. (2002). Growth patterns of craniopharyngiomas in children: role of the diaphragma sellae and its surgical implication. Surg. Neurol. 57, 25-33.

Conflict of Interest Statement: The authors declare that the research was conducted in the absence of any commercial or financial relationships that could be construed as a potential conflict of interest.

Received: 12 November 2011; accepted: 01 February 2012; published online: 20 February 2012

Citation: Ikeda H, Gotoh $\mathrm{H}$ and Watanabe K (2012) Outcome of endoscopyassisted microscopic extended transsphenoidal surgery for suprasellar adult craniopharyngiomas. Front. Endocrin. 3:25. doi: 10.3389/fendo.2012.00025

This article was submitted to Frontiers in Pituitary Endocrinology, a specialty of Frontiers in Endocrinology.

Copyright (c) 2012 Ikeda, Gotoh and Watanabe. This is an open-access article distributed under the terms of the Creative Commons Attribution Non Commercial License, which permits noncommercial use, distribution, and reproduction in other forums, provided the original authors and source are credited. 